\title{
The influence of fibre length and concentration on the properties of glass fibre reinforced polypropylene: 6) the properties of injection moulded long fibre PP at high fibre content.
}

J. L. Thomason, Owens Corning Science \& Technology Centre, s.a., Route de Charneux, B4651 Battice, Belgium

Keywords: A Glass fibres, A Thermoplastic resin, B Mechanical Properties, E Injection moulding

\begin{abstract}
The results of an investigation of the mechanical performance of injection moulded long glass fibre reinforced polypropylene with a glass fibre content in the range $0-73$ weight $\%$ are presented. The composite modulus exhibited a linear dependence on fibre content over the full range of the study. Composite strength and impact resistance exhibited a maximum in performance in the $40-50$ weight $\%$ reinforcement content range. The residual fibre length and fibre orientation in the samples has also been characterised. These parameters were also found to be fibre concentration dependent. Modeling of the composite strength using the measured fibre length and orientation data did enable a maximum in strength to be predicted. However, the position and absolute level of the predicted maximum did not correlate well with the experimental data. Further analysis indicated that deeper investigation of the dependence of the interfacial shear strength and fibre stress at composite failure on the fibre content are required to fully elucidate these results.
\end{abstract}




\section{$\underline{\text { Introduction }}$}

Glass fibre reinforced polypropylene moulding compounds have been available for many years. Since their initial development this class of materials has experienced a rapid growth in their end use applications. This can be attributed to the high level of processing efficiency of such thermoplastic compounds combined with a clean and recyclable nature and an attractive price-performance ratio. However, as is typical with composite materials, we must contend with a balance of processibility to performance. To obtain a high level of processibility with these moulding compounds we must give up a certain level of the reinforcement efficiency of the fibres. Injection moulded composites often contain only relatively short fibres (i.e. shorter than the "critical" fibre length), oriented in complex and often non-optimal patterns. Moreover, extrusion compounding, the classical route for preparing such compounds has a practical processing limit on the maximum fibre content in the range of $40-45$ weight $\%$ of fibres. Therefore the composite applications for these materials cannot be too demanding, where stiffness and strength criteria can be met with fibre volume fractions of 0.2 or less. This is low compared to "high performance" application where volume fractions of 0.5 or greater, of aligned, continuous fibres can be used, usually at the cost of accepting a lower level of processing efficiency.

In the past few years the growth in structural composite usage has resulted in the need for higher output manufacturing processes than have been used previously. This has provided the impetus for the development of techniques to produce long fibre reinforced thermoplastic (and particularly polypropylene) matrix composites which possess both higher performance and mass processibility. The long (but discontinuous) fibre reinforced materials such as Glass Mat Thermoplastic (GMT) and "long-fibre"polypropylene (LF-PP) injection moulding pellets prepared by wire coating, cross head extrusion, or thermoplastic pultrusion techniques, have recently received much attention (1- 11). In particular, the LF or "long-fibre" reinforced pellets for injection moulding can give composites with many significantly enhanced properties in comparison with the more conventional "short-fibre" compounds (11). Much of the attention given to these materials focuses on the effects of fibre length, however due to the aligned nature of the fibres in the LF-PP moulding compounds it is possible to produce pellets with much higher glass fibre contents than those produced with extrusion compounding. The ability to produce moulding compounds with much higher than normal glass contents may enable the production of injection moulded composite components with significantly higher properties than were previously possible. However this would assume that the mechanical properties of these composites continue to increase with increasing fibre content. There are already indications that this may not be the case from data available in the range of $0-40$ weight $\%$ (11). In this paper we present results on a study of the structure-performance relationships in injection moulded LF-PP over a fibre content range of $0-73$ weight $\%$ (0-0.5 volume fraction). We show that, although the composite modulus increases almost linearly across the full range of fibre contents studied, there exists a clear maximum in strength and impact properties. Understanding the structureproperty relationships in injections moulded composites is certainly one of the toughest challenges confronting composite scientists. The microstructure, and consequent 
mechanical performance, of such composites is the result of a complex combination of material and processing parameters. In these composites many of the microstructural parameters have a broad distribution of values and many of these parameters can not be independently varied. A list of some of the parameters which need to be considered in the light of the results that are presented here certainly included fibre length distribution, fibre diameter distribution, fibre orientation distribution, fibre strength distribution, interfacial wetting and adhesion, the presence of voids, changes in matrix properties. In this paper we have also started to address some of the possible reasons behind the observed phenomenon. As is often the case with injection moulded composites the structure-property relationships are complicated and the solution does not lie with any simple explanation. We present here data on the average fibre length and fibre orientation in these composites. In a further paper we will present data on the interfacial shear strength and fibre strain at composite failure.

\section{$\underline{\text { Experimental }}$}

Owens Corning 174C-AD-3000 continuous glass fibres (nominal fibre diameter 20 microns) and Huntsman P4C6Z-059 polypropylene (MFI=35 g/10min) were used to produce LF-PP moulding compounds over a range of glass contents up to 73 weight $\%$ (wt-\%). The level of fibre-matrix interaction in this system was increased by the addition of 2 phr Polybond 3200 coupling agent. The LF-PP moulding compounds were produced by a coating technique similar to that discussed by Bader and Bowyer $(11,12)$. The continuous glass fibre strand was coated using a crosshead die attached to a $50 \mathrm{~mm}$ single screw extruder, which fed the polypropylene with a melt temperature of $230{ }^{\circ} \mathrm{C}$. After water cooling the continuous strand was chopped into pellets of $12.5 \mathrm{~mm}$ length. The compounds were moulded into test bars on a 200-ton Cincinnati Milacron moulding machine. The cylinder heating zones had set point temperatures, rear $180^{\circ} \mathrm{C}$, centre 235 ${ }^{\circ} \mathrm{C}$, front $221{ }^{\circ} \mathrm{C}$, the nozzle temperature was set at $215^{\circ} \mathrm{C}$ and the mould temperature was set at $65^{\circ} \mathrm{C}$. The mould was designed to produce a number of standard test specimens in one shot, all test bars and disks were single end gated.

Unless otherwise stated, all mechanical property testing was performed at $23^{\circ} \mathrm{C}$ and at a relative humidity of $50 \%$. Tensile properties were measured in accordance with the procedures in ASTM D-638, using five ASTM Type I specimens at a crosshead rate of 5 $\mathrm{mm} / \mathrm{min}(0.2 \mathrm{inches} / \mathrm{min})$ and an extensometer gauge length of $50 \mathrm{~mm}$ ( 2 inches). Flexural properties were measured on five specimens in accordance with the procedures in ASTM D-790, at a crosshead rate of $2.5 \mathrm{~mm} / \mathrm{min}(0.1 \mathrm{inches} / \mathrm{min})$ and a span width of $50 \mathrm{~mm}$ ( 2 inches). Izod and modified Charpy impact properties were measured on ten specimens in accordance with the procedures in ASTM D-256 and ASTM D-4812. Multiaxial instrumented impact testing was carried out in accordance with DIN53433 on a Zwick-Rel machine. The injection moulded sample disks were $50 \mathrm{~mm}$ diameter and 3 $\mathrm{mm}$ thick with a support diameter of $40 \mathrm{~mm}$. The impacting tip was $10 \mathrm{~mm}$ diameter with a tip radius of $20 \mathrm{~mm}$, impacting at a constant speed of $1 \mathrm{~m} / \mathrm{s}$. Fibre length and diameters were determined by image analysis and optical microscopy on fibre samples removed from 
the moulded bars after high temperature ashing. The fibre lengths of 500 fibres from each of three test bars were summed to obtain the fibre length distribution for each fibre concentration. Similarly, fibre diameters from 100 fibres from each of three test bars were determined to obtain the fibre diameter distribution. Measurement of fibre orientation was carried out on cross sections of the moulded tensile bars cut perpendicular to the flow direction. The sections were polished and a series of optical micrographs was taken systematically across the thickness of the bar in the centre. Within each photograph all fibres within the central rectangular field of $0.125 \mathrm{~mm} \times 0.18 \mathrm{~mm}$ were analysed. It required 22 fields to traverse the sample cross sections. In the case of samples with a low fibre concentration 44 data fields, equivalent to two passes across the samples crosssection, were used to obtain a sufficient number of fibres. Average values were calculated from these measurements carried out on three test bars from each fibre content. The orientation of any fibre can be determined from its elliptical profile using the equation $(13,14)$

$\cos (\phi)=\mathrm{W} / \mathrm{L}=4 \mathrm{~A} / \pi \mathrm{L}^{2}$

where $\phi$ is the angle the fibre axis makes with the flow direction, $\mathrm{W}$ is the minor axis of the ellipse which should also represent the fibre diameter, $\mathrm{L}$ is the ellipse major axis, and A is the area of the ellipse. Either of possibilities in equation 1 may be used, however it has been shown (14) that the greatest experimental error comes form the measurement of $\mathrm{W}$ and that the area method produces values with a lower degree of uncertainty. If desired the Hermans planar orientation parameter $\left(\mathrm{f}_{\mathrm{p}}\right)$ can be calculated from this data using

$f_{p}=2<\cos ^{2}(\phi)>-1$

where the average value of $\left\langle\cos ^{2} \phi>\right.$ is approximated by

$<\cos ^{2}(\phi)>=\sum_{\mathrm{i}}\left[\mathrm{N}\left(\phi_{\mathrm{i}}\right) \cos ^{2}\left(\phi_{\mathrm{i}}\right)\right] / \sum_{\mathrm{i}}\left[\mathrm{N}\left(\phi_{\mathrm{i}}\right)\right]$

The values of $\mathrm{N}\left(\phi_{\mathrm{i}}\right)$ must first be adjusted (15) by dividing by $\cos \left(\phi_{\mathrm{i}}\right)$ due to the lower probability of the section crossing fibres with higher values of $\phi$.

\section{$\underline{\text { Results }}$}

Figure 1 shows the results for composite tensile and flexural Young's moduli. It can be seen that composite modulus increases with increasing glass fibre content, however there are some interesting differences between the tensile and flexural test results. For the flexural modulus it can be seen that an excellent linear relationship with fibre volume fraction is obtained over the whole range. The Young's modulus determined in tensile testing shows a clear deviation from linearity at higher glass contents. It is also noted that there is a trend for an increase in the variability of the measured value of the Young's modulus at higher reinforcement levels. The data in Figure 2 show that the composite strength does not follow the same linear increase as modulus. Instead there is a continual decrease in reinforcement efficiency as the fibre content increases. Any incremental 
increase in fibre content appears to bring a lower improvement in properties than the previous one. This has been noted and discussed in previous reports $(11,16-18)$. The maximum reinforcement effect is obtained at the 40-50 wt-\% level, above this level the strength of the material is actually decreased by adding more fibre. Indeed, this effect is so significant that by the time we reach $73 \mathrm{wt}-\%$ fibre content we have almost returned to the values of strength for the PP resin alone. The trends for both properties are identical with the data being well fitted by a third order polynomial with a maximum at approximately $40 \mathrm{wt}-\%$. The presence of this maximum in the strength of injection moulded thermoplastics has been implied by extrapolating data in the $0-50 \mathrm{wt}-\%$. range in a number of previous papers. However, this is the first time that we have clearly shown the existence of the maximum. The results for tensile elongation are shown in Figure 3. The addition of even a small fraction of reinforcement dramatically lowers the tensile elongation of the system from a PP resin value of $8.9 \%$. After this initial steep drop tensile elongation further decreases almost linearly with fibre content over the whole 10$73 \mathrm{wt}-\%$ range of this study.

The trends observed in notched impact were practically identical for Izod and Charpy tests, as shown in Figure 4. Indeed, not only do the two test methods show similar trends but also the trends are almost identical to those seen in the composite strength. Initially addition of fibre to the PP leads to a rapid increase in the notched impact resistance. However, this trend reaches a maximum at about $40 \mathrm{wt}-\%$, after which further increase of fibre content leads to a progressively increasing reduction in the Notched Impact until by $73 \mathrm{wt}-\%$ fibre we have almost returned to the original resin value. The results obtained for the unnotched impact resistance are somewhat more complicated as shown in Figure 5. Contrary to most other mechanical properties, semicrystalline thermoplastics often exhibit high levels of unnotched impact resistance which are dramatically lowered by the addition of even small amounts of fibre or filler. If we take this into account along with the fact that the lowest initial level of unnotched impact is reached around $2-4 \mathrm{wt}-\%$. of fibres then the further trends observed are similar to those discussed above. Although the trend lines are somewhat distorted by the high value for the PP resin and the inherent higher levels of scatter obtained in the unnotched impact tests, it does appear that the maximum may actually appear at somewhat lower values (around $30 \mathrm{wt}-\%$ ) for this test in comparison with the strength and notched impact results. The data obtained from the instrumented impact test shown in Figure 6 also follow a similar trend, although in this case the maximum appears to be shifted to the $40-50 \%$ wt range. In Figure 7 it is interesting to note the change in behaviour observed in the unnotched impact trends when we reduce the test temperature to $-40^{\circ} \mathrm{C}$ which is below the glass transition of the PP matrix (approximately $-10{ }^{\circ} \mathrm{C}$ ). The data obtained at $-40{ }^{\circ} \mathrm{C}$ (in a brittle matrix) now show an identical trend as observed with the room temperature data for strength and notched impact, with a maximum obtained at approximately $40 \mathrm{wt}-\%$ fibres. A further interesting point to observe from Figure 7 is that above $40 \mathrm{wt}-\%$ of fibres the Unnotched Charpy impact values appear to be independent of the temperature despite the large difference in the performance of the resin due to the brittle-ductile transition between -40 ${ }^{\circ} \mathrm{C}$ and $23{ }^{\circ} \mathrm{C}$. The results for the heat deflection temperature (HDT) are shown in Figure 8. On addition of fibre the HDT rises rapidly from the resin value to a plateau level of approximately $157^{\circ} \mathrm{C}$ which is close the PP melting temperature of $165^{\circ} \mathrm{C}$. This value is 
fairly constant up to a glass content of approximately $50 \mathrm{wt} \%$. Above this level of fibre content the HDT starts to drop off rapidly although unlike the trends seen in strength and notched impact the level of HDT does not return to the resin only value at the $75 \mathrm{wt}-\%$ level of reinforcement.

In summary of the mechanical performance, the modulus increases linearly across the whole range of glass contents investigated. However, the strength and impact properties all increase initially with glass content but drop off when the glass content is increased above $40 \mathrm{wt}-\%$. Some obvious structural parameters which may be the cause of the poor high glass content performance of these samples are the residual fibre aspect ratio (length/diameter) and fibre orientation factor, both of which have a strong influence on composite performance. We have measured these parameters independently for all samples in this study. The data on fibre length are presented as weight average and number average lengths in Figure 9. It can be seen that the average fibre length in these samples is reduced almost linearly with increasing fibre content. Figure 10 shows the results for the distribution of fibre diameters present in the composites. It can be seen that there exists a fairly wide distribution of fibre diameters which is not uncommon for glass fibre products at these higher micronages (19). The fact that most fibre reinforcements contain a range of fibre diameters is often ignored in micromechanical considerations of composite materials. A recent paper by Thomason revealed that the finer fibres in such a product with a fibre diameter distribution are more susceptible to fibre length reduction during extrusion compounding and injection moulding (19). The average fibre orientation factor (measured on polished composite cross sections) is shown in Figure 11. In this case there appears to be an increase in the average orientation of the fibres parallel to the flow direction as the glass content is increased, although there is a great deal of scatter in the data. By plotting individual the orientation parameter for each field versus position through the thickness of the sample it was possible to obtain some information on the layered structure in the injection moulded samples. However, no significant differences were observed between high and low fibre content samples in this regard.

\section{$\underline{\text { Discussion }}$}

It is well known that the modulus of glass reinforced thermoplastic increases with fibre content. Despite the fact that most practical mouldings are mixed according to weight fractions, analysis of composite properties is normally carried out considering fibre volume fraction since many underlying structure-performance relationships are linear in volume fraction. Fibre volume fraction $\mathrm{V}_{\mathrm{f}}$ is calculated from fibre weight fraction $\mathrm{W}_{\mathrm{f}}$ using the equation

$V_{f}=\left[1+\frac{\rho_{f}}{\rho_{m}} \frac{1-W_{f}}{W_{f}}\right]^{-1}$

which requires both the fibre $\rho_{\mathrm{f}}$ and matrix density $\rho_{\mathrm{m}}$ as input parameters. It is common practice to use the resin density as the matrix density (for PP $905 \mathrm{~kg} / \mathrm{m}^{3}$ ). However, it 
should be realised that there is always a strong possibility that the resin has been modified by the presence of the fibres and that the density of the matrix may be different from that of the resin for a number of reasons (e.g. nucleating effect of the fibres, molecular conformation effects of polymer chains at an interface, effects due to dissolution and reaction of the sizing). Nevertheless, when the tensile modulus is examined as a function of fibre volume fraction for injection moulded glass fibre thermoplastics in the $0-40 \mathrm{wt}-\%$ range an excellent linear relationship is normally observed $(11,16-18)$. The data in Figure 1 can be modelled using a number of approaches. One common approach is to use a simple "rule-of-mixtures" equation

$$
E_{c}=\eta_{0} \eta_{l} V_{f} E_{f}+\left(1-V_{f}\right) E_{m}
$$

We can use the fibre length data reported in Figure 9 to calculate the $\eta_{1}$ factor using the Cox shear lag method (20). Combining these values with the experimental values of composite and matrix modulus we can obtain a value for the orientation parameter $\left(\eta_{\mathrm{o}}\right)$ for each glass content.

Another approach is to use the equation

$$
E_{c}=\eta_{0} E_{1}+\left(1-\eta_{0}\right) E_{2}
$$

where $E_{1}$ and $E_{2}$ are obtained from the Halpin-Tsai equations (21) for the modulus of a unidirectionally reinforced laminate.

$$
E_{j}=E_{m}\left[\frac{1+\xi_{j} \eta_{j} V_{f}}{1-\eta_{j} V_{f}}\right] \quad \eta_{j}=\frac{\left(E_{f} / E_{m}\right)-1}{\left(E_{f} / E_{m}\right)+\xi_{j}} \quad \xi_{1}=\frac{2 L}{D} \quad \xi_{2}=2
$$

In Figure 12 we present results on the calculation of $\eta_{\mathrm{o}}$ using the above two methods and the values of Young's modulus obtained from both tensile and flexural testing. It can be observed the values of $\eta_{\mathrm{o}}$ obtained from equations 5 and 6 are very similar. Furthermore the values obtained from the two test methods are also similar, with the exception of very high glass contents where we also observed a difference in the trends in Figure 1. Given the similarity of the results in Figure 12 and the possible significant potential error in these $\eta_{\mathrm{o}}$ values we feel that it is acceptable to average the values obtained for any given sample. This enables us to compare $\eta_{\mathrm{o}}$ obtained by the optical method and by modulus analysis in Figure 13. It is clear in this Figure that the data are conflicting in terms of the trends for orientation factor versus fibre content observed in Figure 11.

The possible causes for these apparently conflicting results may lie either in errors in the experimental measurements or in the assumptions behind the equations use in the calculation of $\eta_{\mathrm{o}}$. The measurement of the Young's modulus can be assumed to be fairly accurate; however there are a number of issues related to the assumptions made using this method. For instance the assumption that the modulus of the unreinforced resin can be 
substituted for the composite matrix modulus becomes more questionable as the fibre content increases. As discussed above there are numerous mechanisms by which the presence of the fibres can modify the properties of the polymer matrix in their vicinity. Clearly as the fibre content is increased the relative volume fraction of the resin which could be affected by interaction with the fibres increases rapidly. This could consequently lead to an increasing level of error in the calculation of $\eta_{\mathrm{o}}$ using this method. There is also much discussion about the $\mathrm{r} / \mathrm{R}$ factor in the shear lag theory where $\mathrm{r}$ is the fibre radius and $R$ is related to the mean spacing of the fibres. The $r / R$ factor can be related to the fibre volume fraction by assumption of a certain fibre packing arrangement. It is likely that these assumptions may also become more questionable as the fibre content increases. The Halpin-Tsai equations are known to fit some data very well at low fibre volume fractions, but to under-predict some stiffnesses at high volume fractions which might lead to artificially low values for $\eta_{\mathrm{o}}$ at high fibre contents. Tucker and Liang have recently reviewed the assumptions inherent in a number of the models for composite stiffness (22). On the positive side, the modulus method does give a true average for $\eta_{\mathrm{o}}$ over all the fibres in the sample. Conversely, the optical method, like most microscopy techniques, only measures a very small proportion of the fibres in each sample. In our case we examined approximately $1.5 \%$ of the total cross sectional area of the sample. For this reason we feel that for further analysis of the composite mechanical properties the overall averaging of the modulus method gives a more appropriate value for $\eta_{\mathrm{o}}$.

With regard to the composite strength the Kelly-Tyson model for the prediction of the strength $\left(\sigma_{\mathrm{uc}}\right)$ of a polymer composite reinforced with discrete aligned fibres is well known (23).

$$
\sigma_{u c}=\sum_{i}\left[\frac{\tau L_{i} V_{i}}{D}\right]+\sum_{j}\left[\sigma_{f} V_{j}\left(1-\frac{L_{c}}{2 L_{j}}\right)\right]+\left(1-V_{f}\right) \sigma_{u m}
$$

where $\tau$ is the interfacial strength, $V_{i, j}$ the volume fraction of fibres of length $L_{i, j}, \sigma_{f}$ is the fibre strength. The two summation terms arise from the contributions of fibres of sub-critical and super-critical length, where the critical fibre length is defined by $L_{c}=\sigma_{f} D$ / $2 \tau . \sigma_{u m}$ is the matrix strength at the fibre failure strain. For the polypropylene matrix in this study we obtained an excellent fit with the experimental stress-strain curve using $\sigma_{u m}=$ $0.75 \varepsilon^{3}-6.34 \varepsilon^{2}+21.01 \varepsilon$. where $\varepsilon$ is the strain expressed in percent.

There have been few good experimental verifications of this equation due to the, not to be underestimated, complexities of measuring all of the above parameters on a large range of samples. Moreover, the Kelly-Tyson model assumes that all the fibres are aligned in the loading direction which, in practice, rarely occurs and is difficult to achieve experimentally. Injection moulded composites have a complex layered structure with very different average fibre orientation in the different layers $(14,24)$ which the Kelly-Tyson model does not account for. Despite this problem the Kelly-Tyson model is often referred to in relation to the analysis of the strength of discontinuous fibre composites and in particular the calculation of $\mathrm{L}_{\mathrm{c}}$ is often made and quoted as an important parameter. It is possible to fit experimental strength data to this model using a simple numerical orientation factor 
approach. Some care should be exercised when using orientation parameters with the KellyTyson model and it should not be automatically assumed that we might use the same value as used with the various models for modelling composite modulus. For instance for glassfibre polypropylene GMT an excellent fit to experimental tensile strength data (5) was obtained when the fibre contribution in equation 8 was modified by a factor $\eta_{0}=0.2$. However, the GMT laminates have an inplane random fibre orientation for which the orientation parameter for modulus calculation is $\eta_{0}=0.375$. Nevertheless, Thomason has recently published data showing that the Kelly-Tyson model can be reliably used for prediction of the influence of fibre length on the strength of GF-PP both for compression moulded GMT and injection moulded long and short glass reinforcement (11).

We have used the Kelly-Tyson model as a method to investigate whether the measured changes in fibre length and orientation with changing fibre content are sufficient to explain the trends observed in composite strength in Figure 2. By assuming a reasonable value (25) of $\sigma_{\mathrm{f}}=2 \mathrm{GPa}$ and by fitting the data at low glass content values we obtain a value for $\tau=9$ $\mathrm{MPa}$. We then extrapolate to higher glass contents using and these fixed values for $\tau$ and $\sigma_{\mathrm{f}}$. The results of this exercise are shown in Figure 14. We observe that there is a good correlation between the model and experimental data at low glass content due to the use of the fitted value of tau. We can also observe that the model does predict that the changes in fibre length and orientation result in a maximum in composite strength versus glass content. However, this maximum appears at a glass content of approximately $55 \mathrm{wt}-\%$ which is a much higher glass level than the experimental maximum. Moreover, the absolute values predicted for composite strength in the $25-75 \mathrm{wt}-\%$ range are significantly higher than those measured experimentally. It can therefore be concluded that the measured changes in fibre length and orientation are insufficient in themselves to fully explain the experimentally observed trends in composite strength.

In an attempt to gain some further understanding of this problem we have again used equation 8 with the experimental values of fibre length and orientation but now systematically varied one of the parameters, $\tau, \mathrm{D}, \sigma_{\mathrm{f}}$, while keeping the other two constant. For each glass content we have calculated the value of each parameter required to bring $\sigma_{\mathrm{uc}}$ (calculated $)=\sigma_{\mathrm{uc}}$ (experimental). By using this method we hoped to obtain some insight into which of these three parameters might merit further investigation. The results of this exercise are shown in Figure 15. Along the vertical axis in this Figure we observe the value of each of these parameters required to obtain $\sigma_{\mathrm{uc}}$ (calculated $)=\sigma_{\mathrm{uc}}$ (experimental). In order to obtain a clear comparison we have normallised to the fixed value used previously (i.e. $\left.\tau / \tau_{\mathrm{o}}, \sigma_{\mathrm{f}} / \sigma_{\mathrm{f} 0}, \mathrm{Do} / \mathrm{D}\right)$. The results in Figure 15 indicate that the relative change required in these individual parameters to obtain $\sigma_{\mathrm{uc}}$ (calculated) $=\sigma_{\mathrm{uc}}$ (experimental) is the same for all three cases. We have furthermore modelled how these parameter changes would affect the prediction of composite modulus using equations 5 and 7. It is relatively easy to understand how the interfacial shear strength or the fibre stress might be physically reduced in these systems. It is not quite so intuitively obvious how fibre diameter could be increased. However it is an implicit assumption in these models that the fibres are fully dispersed. It is possible to imagine that at higher fibre contents we might get changes in fibre dispersion that could result in fibre bundles, with a higher effective diameter, being present. The higher effective fibre diameter of undispersed fibre bundles 
have been related to changes in the properties of GMT (26). We found that the required increase in the effective fibre diameter at higher glass content resulted in unacceptably large changes (-35\% in modulus at $73 \mathrm{wt}-\%$ glass) in the predicted composite modulus and so this parameter can be eliminated as a variable in further investigations. However, neither the value of $\tau$ or $\sigma_{f}$ are used in the calculation of modulus and so either or both of these parameters should be explored in order to further elucidate the trends observed in Figure 2. The further investigation of these issues will be the subject of a paper in preparation.

\section{Conclusions}

In this investigation of the mechanical performance of injection moulded long glass fibre reinforced polypropylene over a fibre content range of $0-73 \mathrm{wt}-\%$ we have found that composite modulus increase linearly with fibre volume fraction over the whole range of the study. However the trends for strength and impact performance both exhibit nonlinear dependence on the fibre content. Both the strength and notched impact performance show a maximum in performance in the $40-50 \mathrm{wt}-\%$ fibre content range. At higher fibre content these properties decreased significantly and approached the unreinforced polypropylene performance at the highest fibre content of $73 \mathrm{wt}-\%$. The room temperature unnotched impact performance was somewhat more complicated due to the high level exhibited by the unreinforced polypropylene, although there was still clear evidence of a maximum min the $40-50 \mathrm{wt}-\%$ range. Unnotched impact performance at $-40{ }^{\circ} \mathrm{C}$ (below the polymer glass transition temperature) showed the same quadratic type dependence as strength and notched impact. The tensile elongation showed the typical initial steep drop at low fibre content follow by an approximately linear decrease with increasing fibre content.

The residual fibre length in these injection moulded composites decreased linearly with increasing fibre content. The average orientation parameter was also found to be somewhat dependent on fibre content. However we obtained conflicting trends for the orientation parameters depending on whether we obtained the values from optical analysis of polished cross-sections or from analysis of the composite modulus using a rule-of-mixtures equation. By using the measured values of fibre length and orientation in the Kelly-Tyson model to predict composite strength we were able to obtain a curve which did exhibit a maximum. However, the position and absolute level of the predicted maximum did not correlate well with the experimental data. Further analysis using the Kelly-Tyson equation indicated that further investigation of the dependence of the interfacial shear strength and fibre stress at composite failure on the fibre content are required to further elucidate these results. 


\section{List of Figures}

1. Young's modulus versus fibre content (-Tensile, $\boldsymbol{\Delta}$ Flexural, bars show $95 \%$ confidence limits)

2. Strength versus fibre content (๑Tensile, $\boldsymbol{\Delta}$ Flexural)

3. Tensile elongation versus fibre content

4. Notched impact versus fibre content (๑Charpy, $\mathbf{\Delta}$ Izod)

5. Unnotched impact versus fibre content (๑Charpy, $\boldsymbol{\Delta}$ Izod)

6. Instrumented impact versus fibre content

7. Unnotched Charpy impact versus fibre content $\left(-23^{\circ} \mathrm{C}, \boldsymbol{\Delta}-40^{\circ} \mathrm{C}\right)$

8. Heat deflection temperature versus fibre content

9. Residual fibre length versus fibre content ( $\bullet$ weight average, $\boldsymbol{\Delta}$ length average)

10. Fibre diameter distribution of samples in this study

11. Optical orientation parameter versus fibre content

12. Orientation parameter calculated from composite modulus versus fibre content $(\diamond$ Cox Tensile, $\mathbf{c}$ Cox Flexural, $\boldsymbol{\Delta}$ H-T Tensile, $\bullet$ H-T Flexural )

13. Average orientation parameter calculated from composite modulus versus fibre content

14. Tensile strength versus fibre content $(\nabla$ Kelly-Tyson theory, $\boldsymbol{\Delta}$ Experimental)

15. Required change in input parameter to obtain theory=experiment $(\diamond$ IFSS, $\bigcirc$ fibre strength, $\square$ fibre diameter)

\section{$\underline{\text { References }}$}

1. Various Authors, in "Polypropylene: An A-Z reference" Edited by J.KargerKocsis, Kluwer Academic Publishers, London 1999

2. Various Authors, in "Handbook of polypropylene and polypropylene composites" Edited by H.G. Karian, Marcel Dekker, New York 1999

3. Various Authors, in "Polypropylene: Structure, blends and composites" Edited by J.Karger-Kocsis, Chapmann and Hall, London 1995

4. Thomason JL, Vlug MA. The influence of fibre length and concentration on the properties of glass fibre reinforced polypropylene: 1) Tensile and Flexural Modulus. Composites 1996;27A:477-84.

5. Thomason JL, Vlug MA, Schipper G, Krikor HGLT. The influence of fibre length and concentration on the properties of glass fibre reinforced polypropylene: 3) Strength and strain at failure Composites 1996;27A:1075-84.

6. Thomason JL, Vlug MA. The influence of fibre length and concentration on the properties of glass fibre reinforced polypropylene: 4) Impact properties. Composites 1997;28A:277-88.

7. Oelgarth A, Dittmar H, Stockreiter W, Wald HH. GMT or LFT ? A comparison of long-fibre-reinforced thermoplastics. Kunststoffe 1988;88:480-4.

8. Karger-Kocsis J. Swirl mat- and long discontinuous fiber mat-reinforced polypropylene composites - status and future trends. Polym.Compos. 2000;21:514-22. 
9. Yang SW, Chin WK. Mechanical properties of aligned long glass fiber reinforced polypropylene. Polym. Compos. 1999;20:200-15.

10. Chen-Chi MM, Mang-Sang Y, Chen CH, Chiang CL. Processing and properties of pultruded thermoplastic composites. Composites Manufacturing 1990;1:191-6.

11. Thomason JL. The influence of fibre length and concentration on the properties of glass fibre reinforced polypropylene: 5) Injection moulded long and short fibre PP. Composites 2002;33A:1641-52

12. Bader MG, Bowyer WH. An improved method of production of high strength fibre reinforced thermoplastics. Composites 1973;4:150-6

13. Toll S, Andersson PO. Microstructure of long- and short-fibre reinforced injection moulded polyamide. Polym.Composites 1993;14:116-25.

14. Toll S, Andersson PO. Microstructural characterization of injection moulded composites using image analysis. Composites 1991;22:298-306.

15. Bay RS, Tucker CL. Stereological measurement and error estimates for threedimensional fiber orientation. Polym. Eng. Sci. 1992;32:240-53.

16. Thomason JL. Micromechanical Parameters from Macromechanical Measurements on Glass Reinforced Polypropylene. Compos.Sci.Technol. 2002;62:1455-68.

17. Thomason JL. Micromechanical Parameters from Macromechanical Measurements on Glass Reinforced Polyamide 6,6. Compos.Sci.Technol. 2001;6:2007-16.

18. Thomason JL. Micromechanical Parameters from Macromechanical Measurements on Glass Reinforced Polybutyleneterepthalate. Composites Part A 2001;33:331-39

19. Thomason JL. The influence of fibre properties of the performance of glass-fibrereinforced polyamide 6,6. Compos.Sci.Technol. 1999;59:2315-28.

20. Cox HL. The elasticity and strength of paper and other fibrous materials. Brit.J.Appl.Phys. 1952;3:72-9.

21. Halpin JC, Kardos JL. The Halpin-Tsai equations: A review. Polym.Eng.Sci. 1976;16:344-52.

22. Tucker CL, Liang E. Stiffness predictions for unidirectional short-fiber composites: Review and evaluation. Compos.Sci.Technol. 1999;59:655-71.

23. Kelly A, Tyson WR. Tensile properties of fibre-reinforced metals. J.Mech.Phys.Solids, 1965;13:329-50.

24. Akay M., Barkley D. Fibre orientation and mechanical behaviour in reinforced thermoplastic injection mouldings. J.Mater. Sci. 1991;26:2731-42

25. Thomason JL, Kalinka G. A technique for the measurement of reinforcement fibre tensile strength at sub-millimetre gauge lengths. Composites Part A 2001;33:85-90.

26. Ericson M, Berglund L. Deformation and fracture of glass-mat-reinforced polypropylene. Compos.Sci.Technol. 1992;43:269-81. 

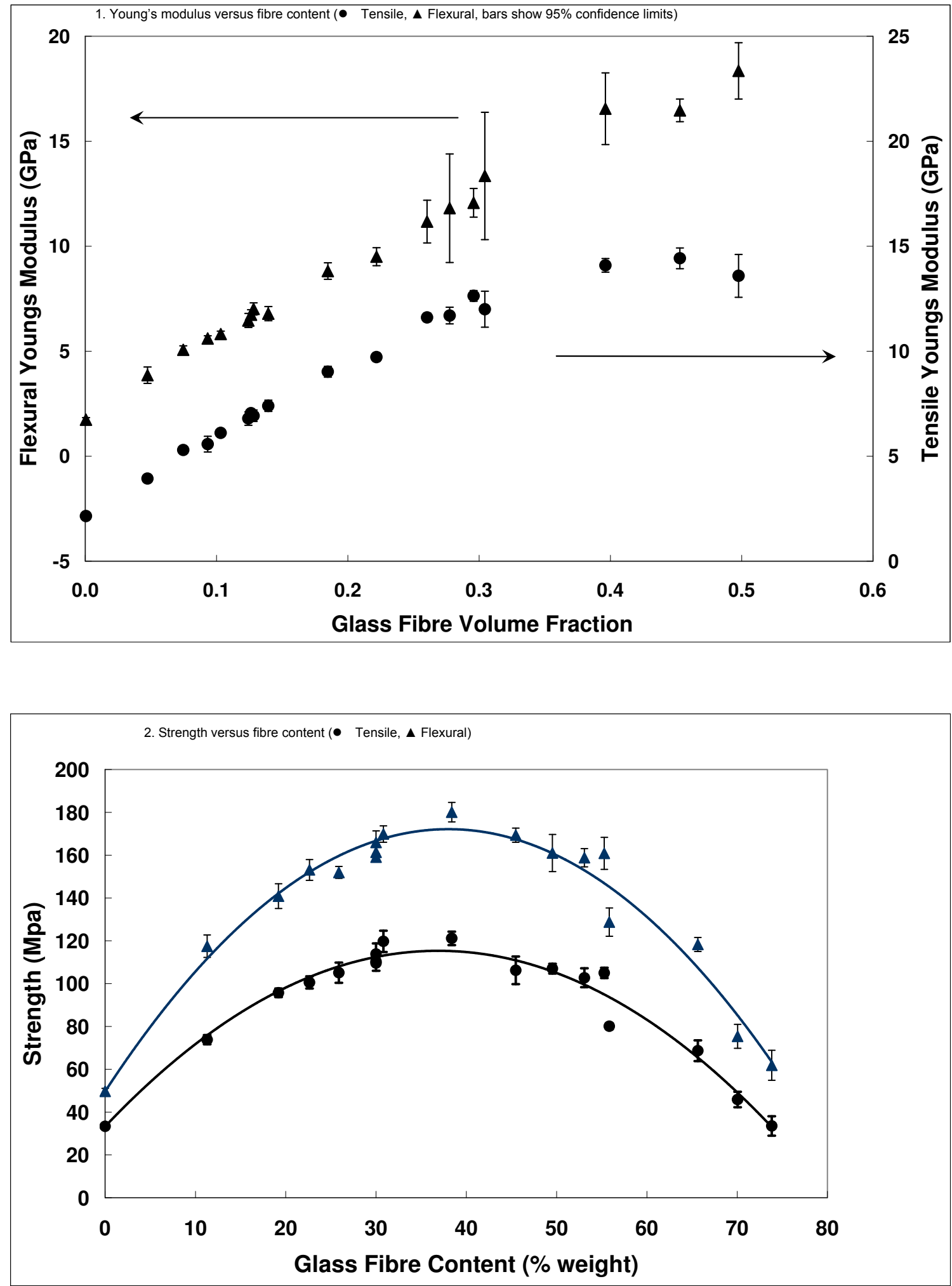

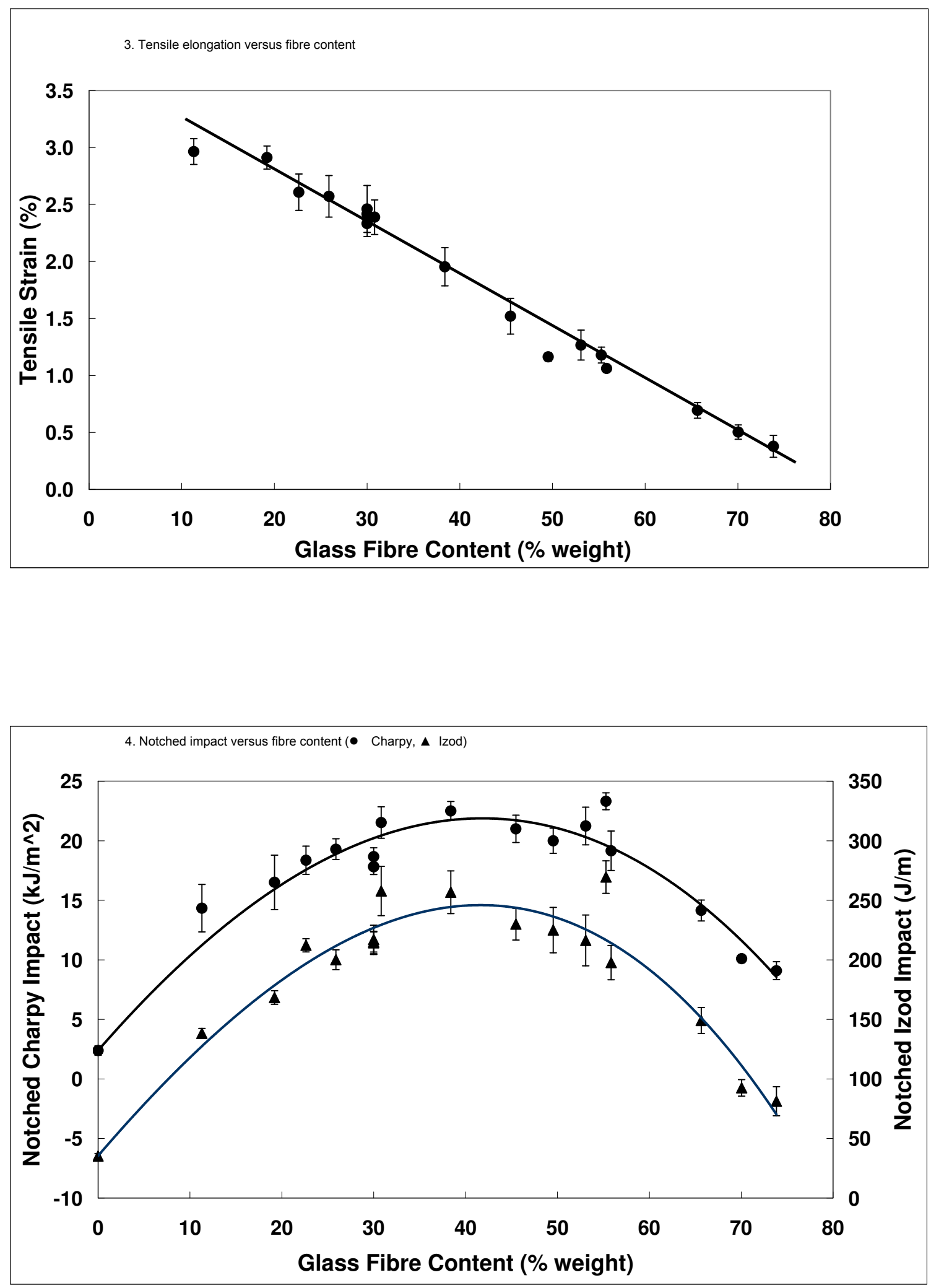

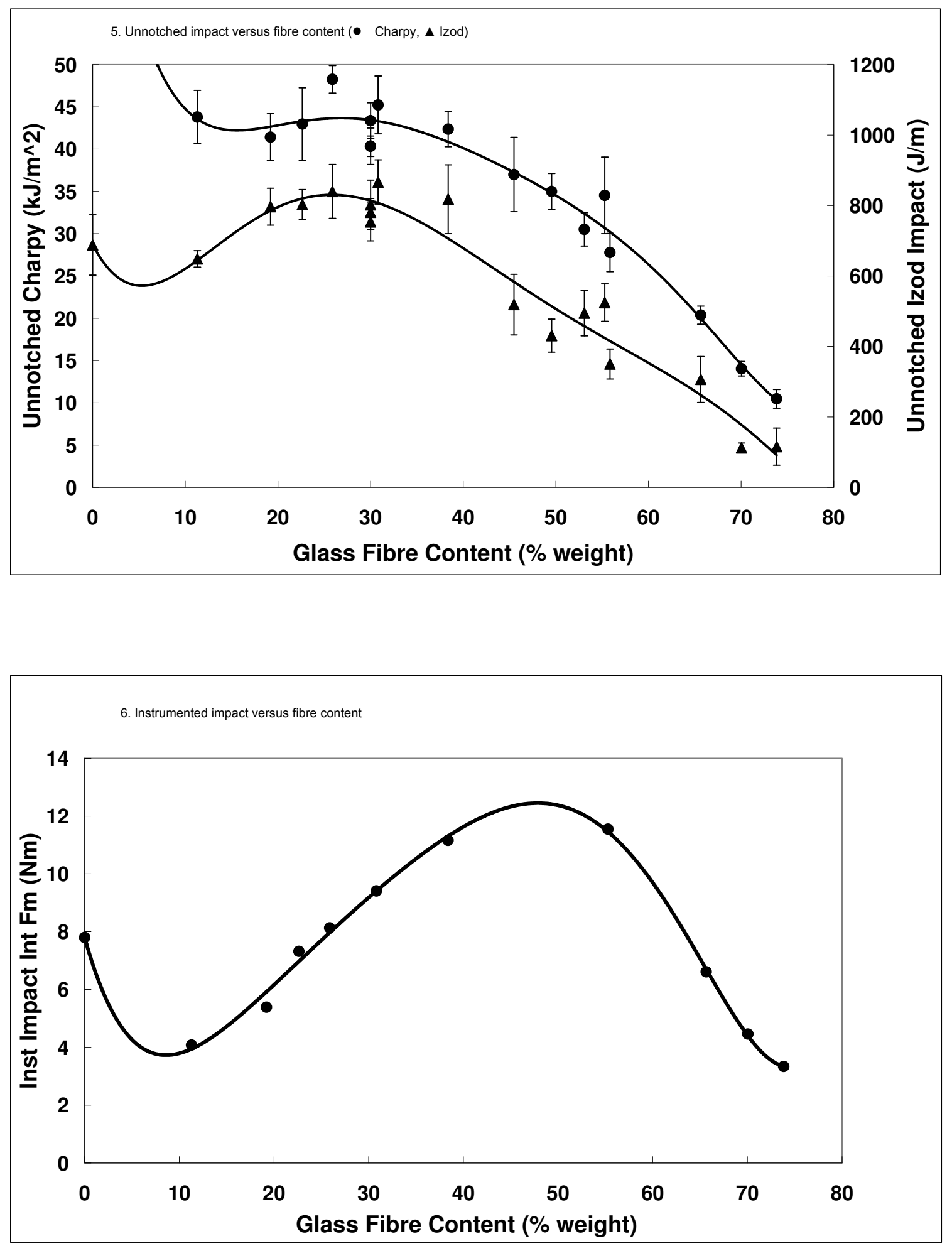

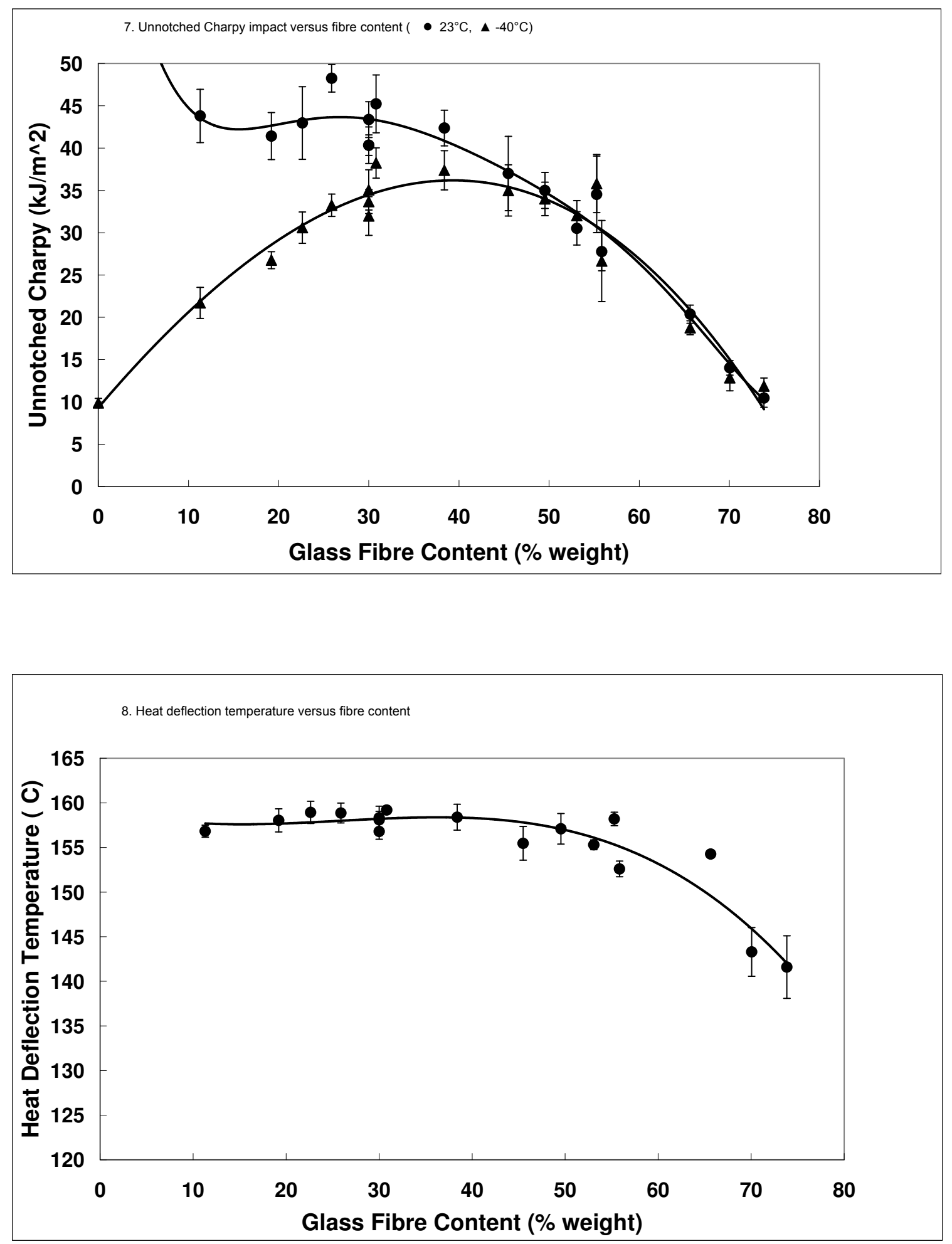

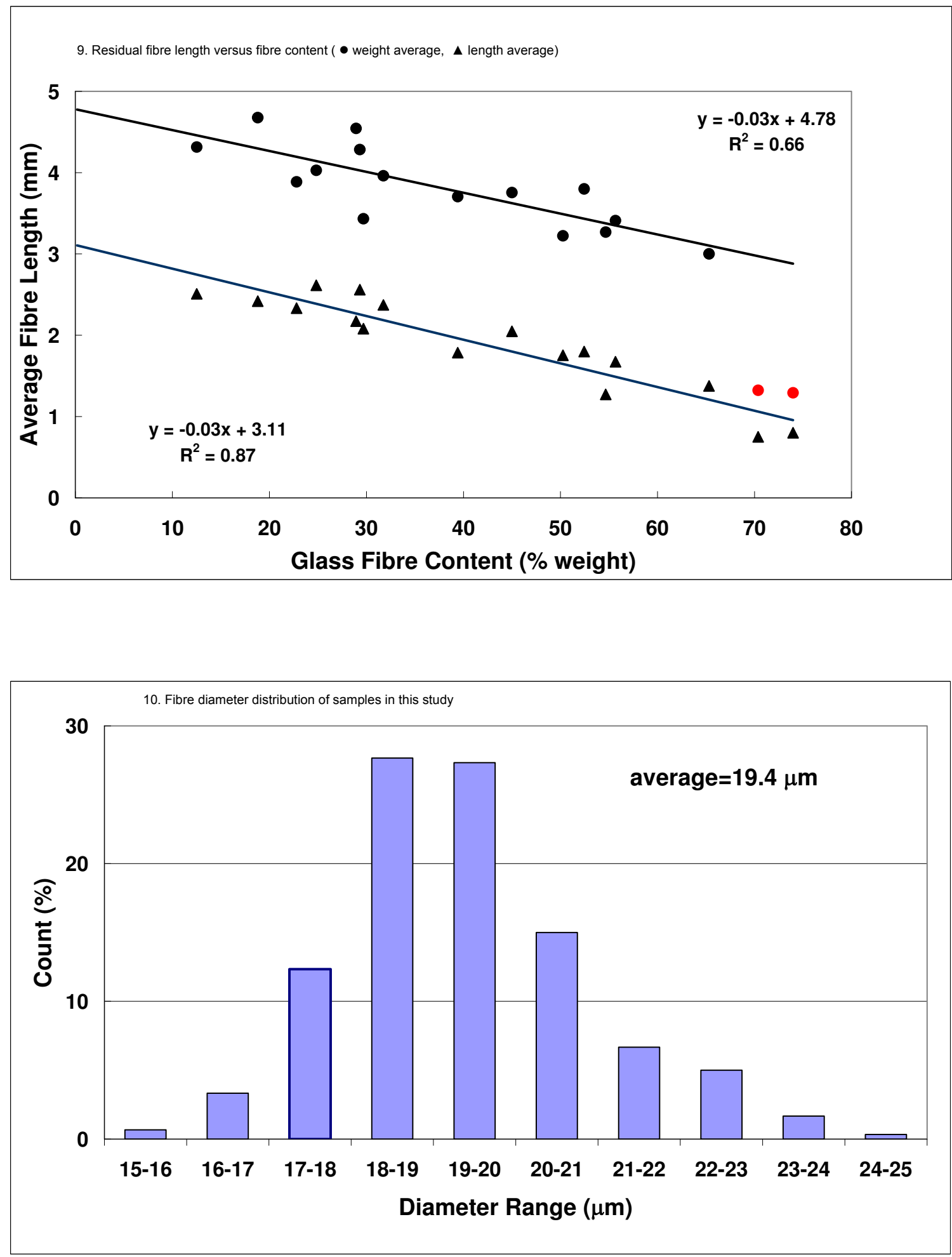

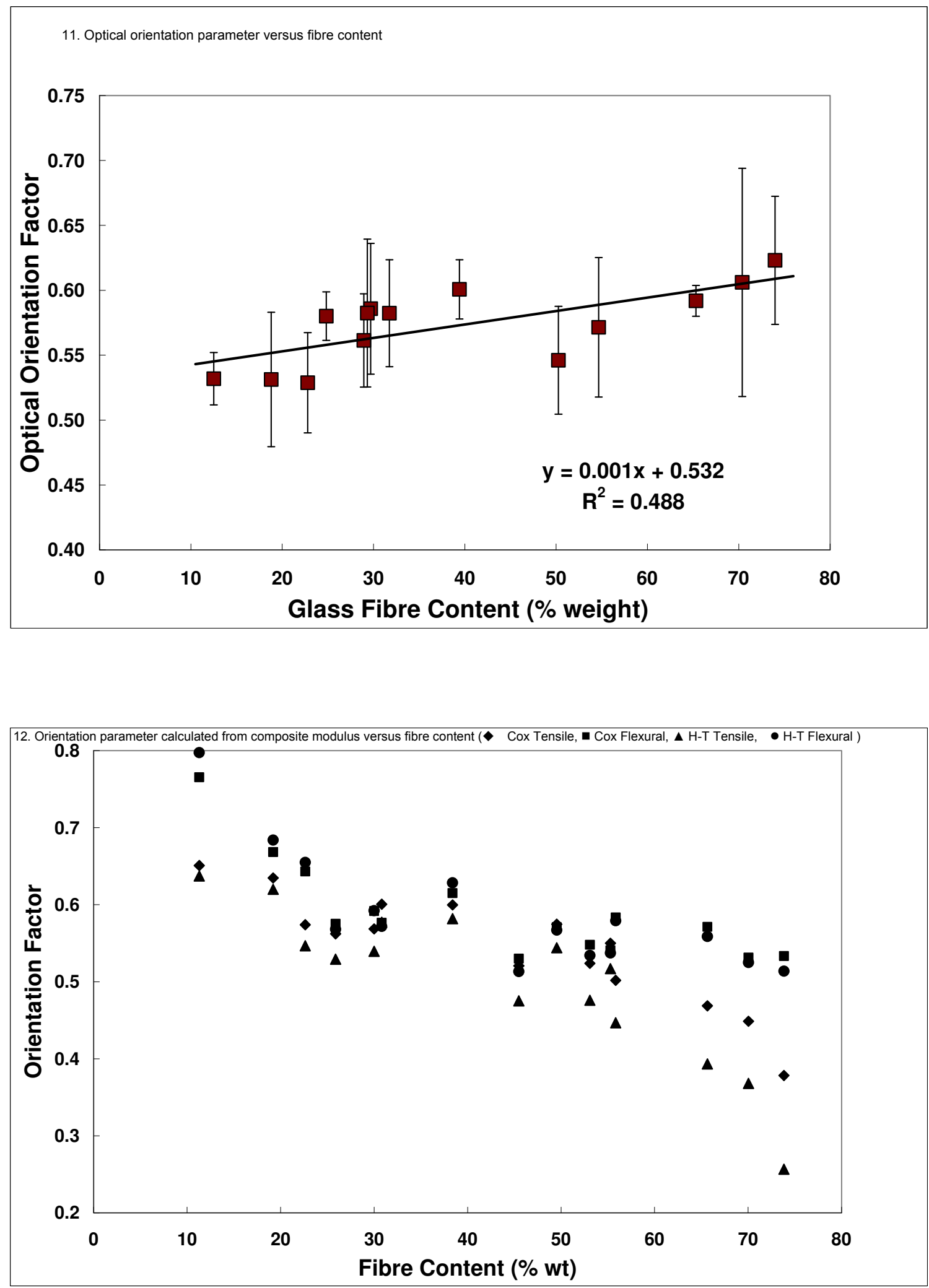

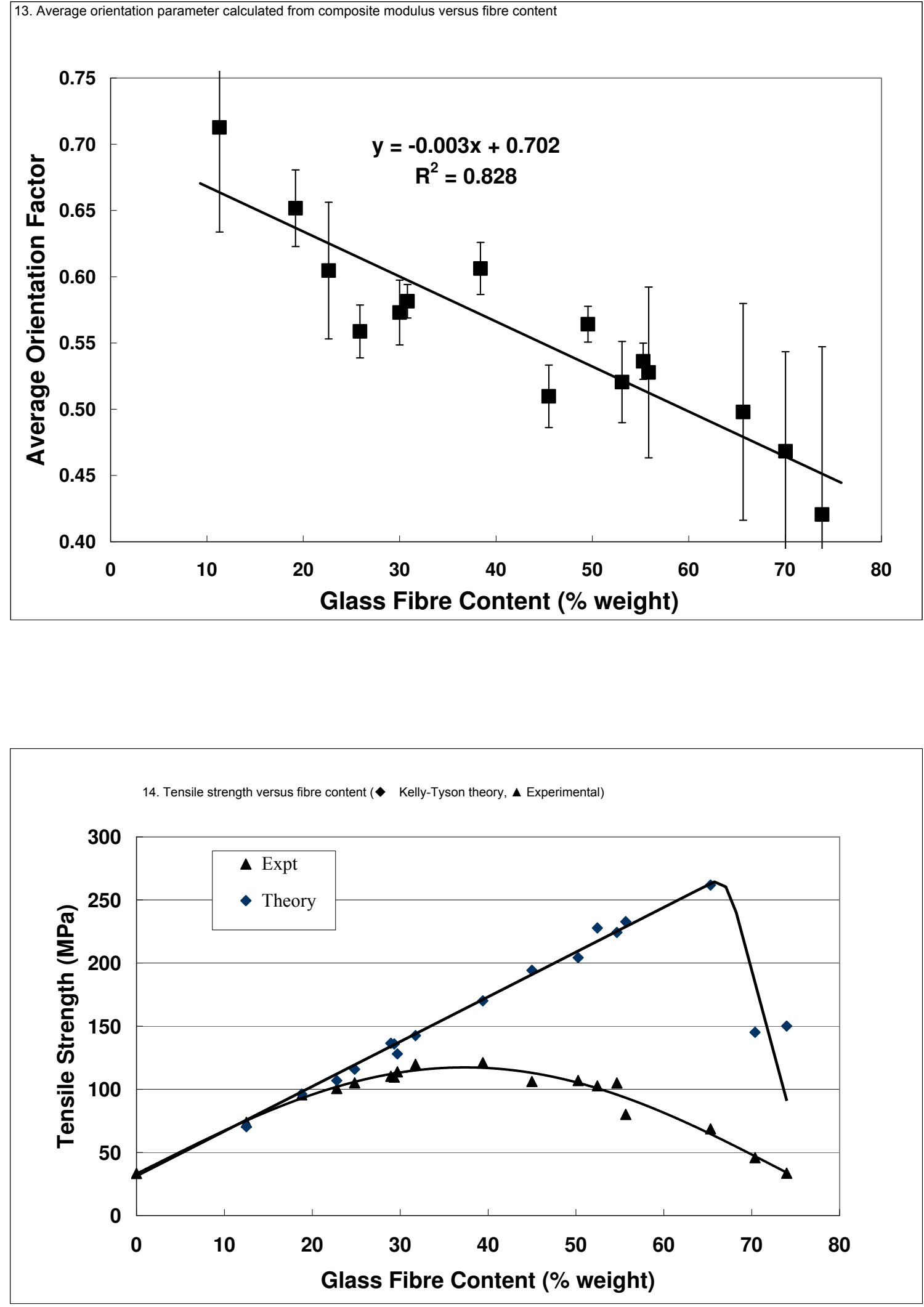


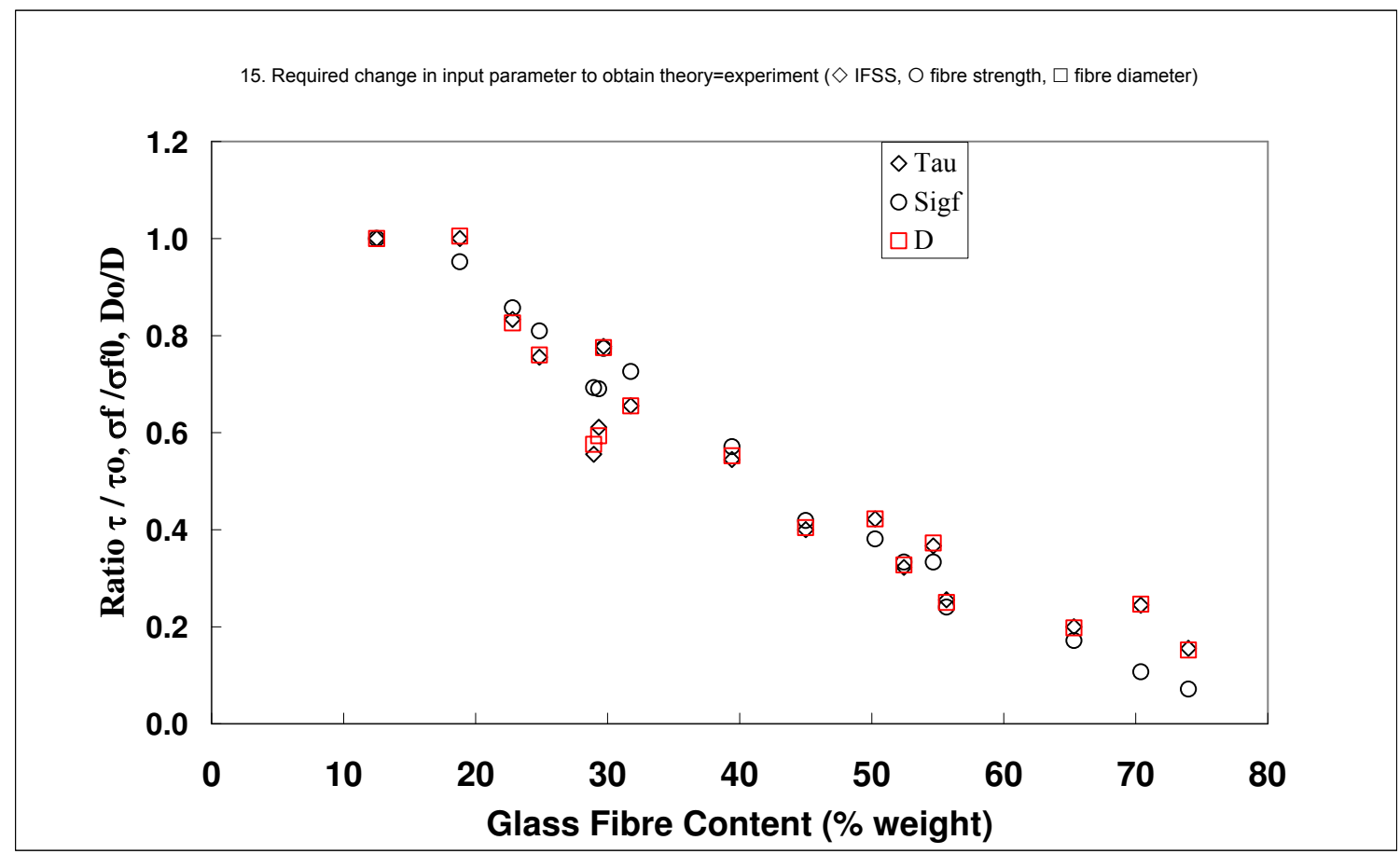

